\title{
Comparison and evaluation of the reliability of indexes of adherence to the Mediterranean diet
}

\author{
Raimon Milà-Villarroel ${ }^{1}$, Anna Bach-Faig ${ }^{2}$, Josep Puig ${ }^{1}$, Anna Puchal ${ }^{3}$, Andreu Farran ${ }^{4}$, \\ Lluis Serra-Majem ${ }^{2,5}$ and Josep Lluis Carrasco ${ }^{1, *}$ \\ 'Biostatistics, Faculty of Medicine, Department of Public Health, University of Barcelona, Casanova Street 143, \\ 08036 Barcelona, Spain: ${ }^{2}$ Mediterranean Diet Foundation, Barcelona, Spain: ${ }^{3}$ Center for Superior Studies on \\ Human Nutrition and Dietetics (CESNID), University of Barcelona, Barcelona, Spain: ${ }^{4}$ Department of Nutrition \\ and Bromatology, University of Barcelona, Barcelona, Spain: ${ }^{5}$ Department of Clinical Sciences, University of \\ Las Palmas de Gran Canaria, Spain
}

Submitted 13 May 2011: Accepted 7 September 2011

\begin{abstract}
Objective: To compare and evaluate the reliability of several indexes of adherence to the Mediterranean diet.

Design: The ten indexes included in the analysis were: Mediterranean Diet Score (MDS), Mediterranean Score (MS), Dietary Score (DS), Mediterranean-Dietary Quality Index (Med-DQI), Mediterranean Dietary Pattern adherence index (MDP), Mediterranean Adequacy Index (MAI), Mediterranean Style Dietary Pattern Score (MSDPS), Mediterranean food pattern PREDIMED Study (MeDiet-PREDIMED), relative Mediterranean diet (rMED) and Cardioprotective Mediterranean diet index. Factor analysis using the correlations between indexes was applied. The correlation with factors and the reliability coefficient were calculated.

Setting: A total of 324 healthy undergraduates at the University of Barcelona, Spain, were surveyed.

Results: The highest correlations were observed between MDP adherence index and MAI (0.82); MAI and MSDPS (0.80); and MDS and rMED (0.77). Factor analysis showed a hidden common factor that explained over $70 \%$ of the variability (71.03\%). This factor is understood as 'adherence to the Mediterranean diet'. The indexes that showed the highest correlation with this factor were Med-DQI $(0 \cdot 85)$, MDS $(0 \cdot 84)$, rMED $(0 \cdot 80)$ and MAI $(0 \cdot 80)$. These indexes showed acceptable performance in measuring the adherence to the Mediterranean diet. The components that correlated strongly with this factor were monounsaturated-to-saturated fatty acid ratio (MS ratio), fruit and vegetables. Furthermore, a second common factor was found explaining $18 \%$ of the variability. This second factor is highly positive related to dairy products and lean meat, and negative related to MS ratio.

Conclusions: The indexes showed satisfactory performance in assessing adherence to the Mediterranean diet. However, in order to improve the reliability and concordance between the indexes, further studies are required to select the components, the number of components, and the scoring criteria of the indexes to improve their internal consistency.
\end{abstract}

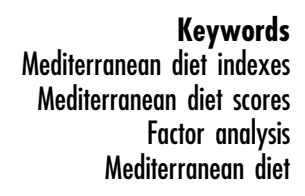

Keys and Grande Covian ${ }^{(1)}$ introduced the concept of the Mediterranean diet in the 1950s. Initially describing eating habits in the Mediterranean area, this concept has further been referred to as the traditional dietary pattern found in the olive-growing regions along the Mediterranean coastline in the late 1950 s and early $1960 \mathrm{~s}^{(1)}$. The traditional Mediterranean diet is characterised by a high intake of vegetables, legumes, fruits and nuts, unrefined cereals and olive oil (but a low intake of saturated lipids); a moderate intake of fish; a low-to-moderate intake of dairy products; a low intake of meat and poultry and a regular but moderate intake of ethanol, mainly in the form of wine and generally during meals ${ }^{(2)}$. However, this diet is not homogeneous as there are regional variations, influenced by various factors, such as sociocultural, religious and economic determinants ${ }^{(3,4)}$.

The Mediterranean dietary pattern has consistently been shown to provide a degree of protection against CVD and major chronic degenerative diseases ${ }^{(5,6)}$. Research in this field over recent years has focused on estimating adherence to the Mediterranean diet rather than analysing the individual components of the diet in 
relation to the health of the population ${ }^{(7-10)}$. However, no single direct method to quantify and assess adherence is available. Consequently, a large number of indirect indexes have been proposed ${ }^{(10)}$. Most Mediterranean diet scores have been developed using dietary patterns defined a priori. These indexes summarise the diet by means of a single score that results from a function of numerous components (previously selected on the basis of prior knowledge or scientific evidence), such as food, food groups or a combination of foods and nutrients ${ }^{(10)}$. The validity of these indexes has been evaluated by examining their relationship with nutrient adequacy and several health outcomes (i.e. nutrient-related diseases). For instance, indexes evaluating adherence to the Mediterranean diet have been successfully applied to studies on life expectancy ${ }^{(7,8,11)}$, cardiovascular risk $^{(12-14)}$, cancer $^{(15,16)}$, hypertension ${ }^{(17)}$, obesity ${ }^{(18,19)}$ and diabetes ${ }^{(20)}$

Nevertheless, recent publications have concluded that some indexes do not provide a significantly more reliable predictive capacity for disease or mortality than individual dietary factors ${ }^{(21)}$. This observation could be attributed to the distinct ways in which each index has been developed. In this regard, indexes show great variation in the following: the number of components (nutrients, foods or food groups intake); classification categories for each subject; measurement scales; statistical parameters (mean, median or tertiles of daily intake); and the contribution of each component (positive or negative) to the total score ${ }^{(0,22)}$. Thus, here we compared and evaluated in the same sample the reliability of several indexes used to measure adherence to the Mediterranean diet.

\section{Material and methods}

\section{Subjects}

The survey was carried out on 336 healthy undergraduates. Students were invited to participate in the study during their degree course. Data were collected over four consecutive academic years (2003-2007) from students registered in the third year of Human and Dietetics Studies at the University of Barcelona, Spain. After exclusion of errors or inconsistencies in data and incomplete questionnaires, the final sample included 324 subjects. Information on food consumption was collected through a quantitative FFQ.

\section{FFQ}

Nutrient and food intake was estimated using an adaptation of the quantitative FFQ used in the Catalonian nutritional survey ${ }^{(23)}$. This form includes approximately fifty-one commonly consumed food and beverage items. The questionnaire was administered in person by trained interviewers. Standard portion sizes were used to estimate the amounts consumed, and nutrient and ethanol intakes were calculated using the food composition database of the Centre for Superior Studies on Nutrition and Dietetics
(CESNID; University of Barcelona) ${ }^{(24)}$. For each participant, we calculated intake in $\mathrm{g} / \mathrm{d}$ of various food groups and nutrients, as well as total energy intake.

We focused on each food and nutrient variable included in general indexes used to assess observance of the Mediterranean diet. The most common food groups used were as follows: pulses; cereals; potatoes; fruit; vegetables; fish and seafood; olive oil; alcohol; red meat; dairy products; cereals; monounsaturated-to-saturated fatty acid ratio (MS ratio); and lean meat (poultry and rabbit).

\section{Indexes of adberence to the Mediterranean diet}

For the purpose of the present study, ten indexes were included in the analysis: Mediterranean Diet Score $(\mathrm{MDS})^{(25)}$, Mediterranean Score (MS) $)^{(26)}$, Dietary Score $(\mathrm{DS})^{(27)}$, Mediterranean-Dietary Quality Index (Med-DQI) ${ }^{(28)}$, Mediterranean Dietary Pattern adherence index (MDP) ${ }^{(29)}$, Mediterranean Adequacy Index (MAI) ${ }^{(30)}$, Mediterranean Style Dietary Pattern Score (MSDPS) ${ }^{(31)}$, Mediterranean food pattern PREDIMED Study (MeDiet-PREDIMED) ${ }^{(32)}$, relative Mediterranean diet (rMED) ${ }^{(33)}$ and Cardioprotective Mediterranean diet index $\left(\right.$ Cardio) ${ }^{(34)}$.

\section{Statistical analyses}

To describe the data, mean values and standard deviation were calculated and, on the basis of their quartile values, indexes were classified into four groups. As the indexes differ in the scales used to assess adherence, the association between them was evaluated by means of Spearman's coefficient of correlation.

Given that the attribute 'adherence to the Mediterranean diet' cannot be measured directly (latent attribute), it is necessary to use indirect measures obtained from the indexes. It was therefore assumed that all the indexes were designed to measure this attribute, although they may differ in the definition of the Mediterranean dietary pattern. Thus, we performed a factor analysis ${ }^{(35)}$ using the correlations between indexes as data. We used the goodness-of-fit index $(\mathrm{GIF})^{(35)}$ to assess the degree of relationship between factors and indexes. The GIF can be interpreted as the percentage of variability of indexes explained by the model. The factor model that better fit the data was selected using the Akaike Information Criterion (AIC). The correlation with factors and the reliability coefficient are provided for each index. All analyses were performed using the SAS statistical software package version 9·1 (SAS Institute, Cary, NC, USA).

\section{Results}

A total of 324 FFQ were used to calculate the ten adherence indexes. The mean, minimum, maximum and percentile (25th, 50th and 75th) values of each index are shown in Table 1. The DS index showed the highest value of adherence to the Mediterranean diet, whereas the other indexes centred on the average value. 
A first comparison of indexes was made by grouping them into four categories on the basis of their theoretical range of scores and comparing the frequencies of each category. Most indexes accumulated the highest scores in central values, and the second and third categories (54\% and 25\%, respectively), except DS and MSDPS, which resulted in more extreme values (Fig. 1). Most of the values from the MSDPS index fell in the first two ranges, with 159 $(49 \cdot 1 \%)$ and $165(50 \cdot 9 \%)$ subjects in ranges 1 and 2,

Table 1 Descriptive analysis of indexes of adherence to the Mediterranean diet

\begin{tabular}{|c|c|c|c|c|c|c|}
\hline Indexes & Mean & Min & Max & Pctl25 & Pct|50 & Pctl75 \\
\hline MDS & $4 \cdot 75$ & 0.00 & 9.00 & 3.00 & $5 \cdot 00$ & $6 \cdot 00$ \\
\hline MS & $18 \cdot 82$ & $8 \cdot 00$ & $28 \cdot 00$ & $17 \cdot 00$ & $19 \cdot 00$ & $21 \cdot 00$ \\
\hline DS & $43 \cdot 78$ & $30 \cdot 00$ & $50 \cdot 00$ & $42 \cdot 00$ & $44 \cdot 00$ & $46 \cdot 00$ \\
\hline Med-DQI & $7 \cdot 33$ & $2 \cdot 00$ & $12 \cdot 00$ & $6 \cdot 00$ & $7 \cdot 00$ & $9 \cdot 00$ \\
\hline MAI & $1 \cdot 67$ & 0.29 & $7 \cdot 36$ & $1 \cdot 04$ & $1 \cdot 48$ & 1.95 \\
\hline MDP & $40 \cdot 95$ & 0.00 & $100 \cdot 00$ & $33 \cdot 38$ & $40 \cdot 87$ & $48 \cdot 39$ \\
\hline rMED & $8 \cdot 06$ & $2 \cdot 00$ & $16 \cdot 00$ & $6 \cdot 00$ & $8 \cdot 00$ & $10 \cdot 00$ \\
\hline MSDPS & $25 \cdot 17$ & $7 \cdot 94$ & $44 \cdot 61$ & $19 \cdot 82$ & $25 \cdot 33$ & $30 \cdot 58$ \\
\hline PREDIMED & 5.93 & $2 \cdot 00$ & $10 \cdot 00$ & $5 \cdot 00$ & $6 \cdot 00$ & $7 \cdot 00$ \\
\hline Cardio & $4 \cdot 16$ & $1 \cdot 00$ & $7 \cdot 00$ & 3.00 & 5.00 & 5.00 \\
\hline
\end{tabular}

Min, minimum; Max, maximum; Pctl25, 25th percentile; Pctl50, 50th percentile; Pctl75, 75th percentile; MDS, Mediterranean Diet Score; MS, Mediterranean Score; DS, Dietary Score; Med-DQI, Mediterranean-Dietary Quality Index; MAI, Mediterranean Adequacy Index; MDP, Mediterranean Dietary Pattern adherence index; rMED, relative Mediterranean diet; MSDPS, Mediterranean Style Dietary Pattern Score; PREDIMED, Mediterranean food pattern PREDIMED Study; Cardio, Cardioprotective Mediterranean diet index. respectively. In contrast, the values from the DS index concentrated in the ranges 3 and 4 , but especially in the latter (78\%; 253 subjects).

Table 2 shows the correlations among indexes. Only three correlations were high. The highest correlations (dark grey) were observed between MDP adherence index and MAI (0.82); MAI and MSDPS (0.80); and MDS and rMED $(0 \cdot 77)$. The remaining correlations were fair $(0 \cdot 5-0 \cdot 7$; light grey) or poor $(<0 \cdot 5$; white $)$. More than half of the indexes show poor correlation.

Factor analysis showed a common factor 1, which explained more than $70 \%$ of the variability (GIF: $71 \cdot 03 \%$; AIC: $512 \cdot 43$; Table 3). Thus, this would be the main common factor between indexes and can be understood as 'adherence to the Mediterranean diet'. The indexes that most correlated with this factor were Med-DQI $(0 \cdot 85)$, MDS (0.83), rMED $(0 \cdot 80)$ and MAI $(0 \cdot 83)$, all of which showed satisfactory performance in measuring adherence to the Mediterranean diet. However, $30 \%$ of the variability was attributed to measurement error, that is, variability between indexes that is not caused by the 'adherence to the Mediterranean diet' factor. The MS, DS and Cardio indexes showed less accuracy in measuring this factor, with correlations of $0 \cdot 67,0.64$ and $0 \cdot 67$, respectively.

We next fitted a model with two factors. This model showed an increase in explained variance and a better fit of data (GIF: $88 \cdot 97 \%$; AIC: $160 \cdot 08$ ). Thus, a second common factor was observed to explain $18 \%$ of the
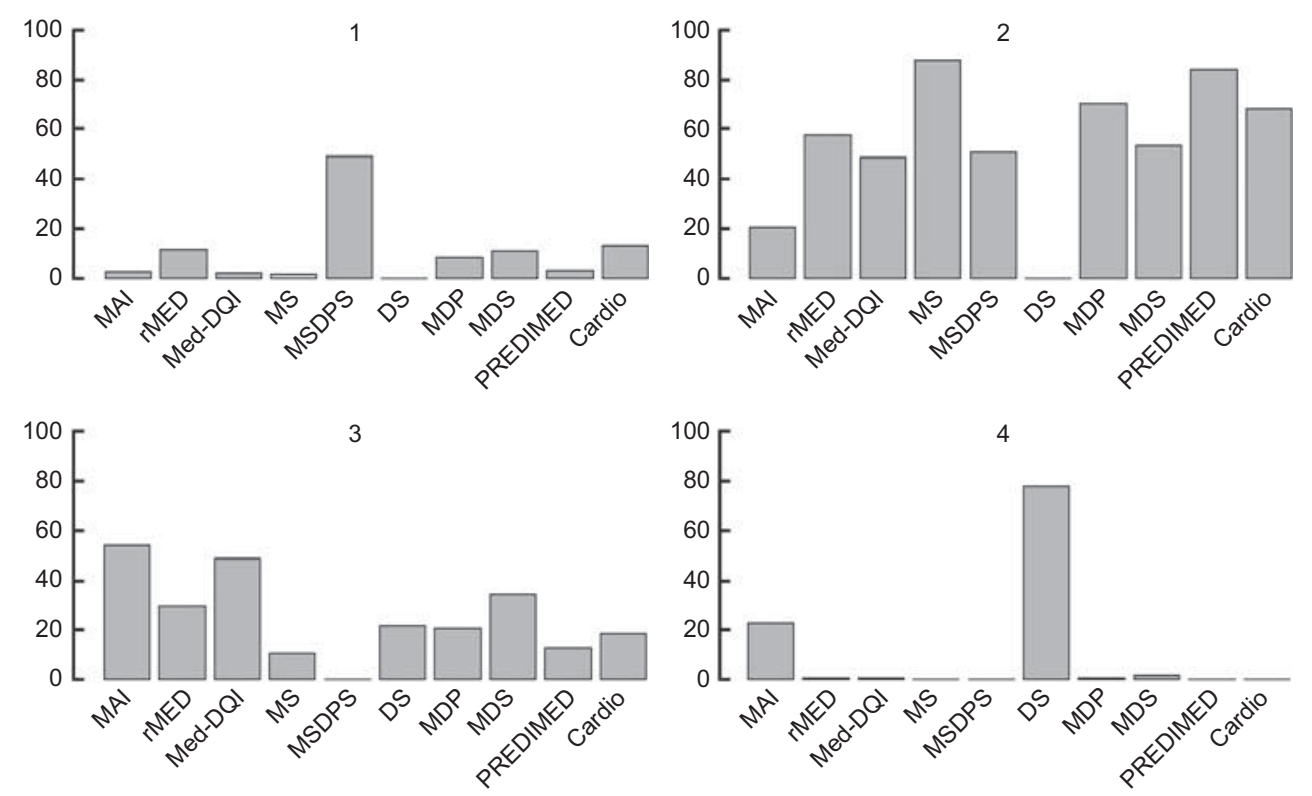

Fig. 1 Comparison of indexes according to the frequencies of each category. The reference values used were: Mediterranean Adequacy Index (MAI): $1(<0,5), 2(0,5-1), 3(>1-2)$ and $4(>2)$; relative Mediterranean diet (rMED): 1 (0-4), 2 (5-9), 3 (10-14) and 4 (15-18); Mediterranean-Dietary Quality Index (Med-DQI): 1 (0-3), 2 (4-7), 3 (8-11) and 4 (11-14); Mediterranean Score (MS): 1 (0-11), 2 (12-22), 3 (23-33) and 4 (34-44); Mediterranean Style Dietary Pattern Score (MSDPS): 1 (0-25), 2 (>25-50), 3 (>50-75) and 4 (>75-100); Dietary Score (DS): 1 (0-13), 2 (14-27), 3 (28-41) and 4 (42-55); Mediterranean Dietary Pattern adherence index (MDP): 1 (0-25), 2 (>25-50), 3 (>50-75) and 4 (>75-100); Mediterranean Diet Score (MDS): 1 (0-2), 2 (3-5), 3 (6-8) and 4 (9-10); Mediterranean food pattern PREDIMED Study (MeDiet-PREDIMED): 1 (0-3), 2 (4-7), 3 (8-10) and 4 (11-14); Cardioprotective Mediterranean diet index (Cardio): 1 (0-2), 2 (3-5), 3 (6-7) and 4 (8-9) 
variability (Table 3). This factor allowed us to identify three groups of indexes: group 1 (MAI, MDP-AI and MSDPS), group 2 (MDS, PREDIMED, rMED and Med-DQI) and group 3 (DS, Cardio and MS) (Fig. 2). Correlations between indexes and the second factor ranged from -0.52 (MAI), -0.37 (MSDPS) and -0.35 (MDP) to 0.45 (DS). The remaining indexes showed correlations from $0 \cdot 02$ to $0 \cdot 28$, except for MED-DQI and rMED, which were independent for this second factor (correlation close to 0 ).

In order to interpret the meaning of this second factor, we used the main components of the food indexes (pulses, cereals and potatoes, fruit, vegetables, fish, alcohol, red meat, dairy products, MS ratio, olive oil and lean meat (poultry and rabbit meat) to compute correlations with the factor scores. The components that highly correlated with the first factor (i.e. 'adherence to the Mediterranean diet') were MS ratio, vegetables and fruits. This observation confirms the hypothesis that the first factor is the 'adherence to the Mediterranean diet' attribute. In contrast, the second factor was positively correlated to dairy products and lean meat, and negatively correlated to MS ratio (Table 4).

\section{Discussion}

The study of dietary patterns using diet quality indexes to examine interactions between dietary components and their effect on several health parameters is recommended over the study of single dietary components ${ }^{(36,37)}$. Given the documented protective effects of the Mediterranean diet against several diseases ${ }^{(6-8)}$, recent years have witnessed greater attention on indexes that assess adherence to this diet. The usefulness of Mediterranean diet adherence indexes has been widely demonstrated ${ }^{(10)}$. Furthermore, they have also been reviewed and validated in relation to a number of health parameters (cancer, obesity, mortality, etc. ${ }^{(15,16,18,20)}$. However, little attention has been given to the way in which these indexes are developed (cut-off points, adjustments for energy and nutrients, reference populations, adjustment for confusion variables). Moreover, the absence of common criteria to identify the components that constitute the Mediterranean dietary pattern hinders the development of these indexes (each index includes the components considered most suitable depending on the study objectives and data available) and comparisons.

Here we compared ten indexes of adherence to the Mediterranean dietary pattern in order to assess their reliability. Although these indexes could have been validated in other independent studies, we are testing them all in one sample. Most of the indexes (except DS and MSDPS) did not use their whole range of measurement, and hence this implies greater difficulty in discriminating individuals on the basis of their compliance with the Mediterranean diet (Table 1). Thus, depending on the index used, the study population presents an extremely variable degree of adherence. For example, when the DS 
Table 3 Correlation among factors ( 1 and 2$)$ and indexes of adherence to the Mediterranean diet

\begin{tabular}{|c|c|c|c|c|c|c|c|c|}
\hline \multirow[b]{2}{*}{ Index } & \multicolumn{3}{|c|}{ Factor 1} & \multicolumn{3}{|c|}{ Factor 2} & \multirow[b]{2}{*}{ Variance error } & \multirow[b]{2}{*}{ SE } \\
\hline & $\beta$ & $\mathrm{SE}$ & $R^{2}(\%)$ & $\beta$ & SE & $R^{2}(\%)$ & & \\
\hline MDS & 0.83 & 0.048 & 69 & $0 \cdot 17$ & 0.033 & 3 & 0.25 & 0.028 \\
\hline MS & 0.67 & 0.049 & 45 & $0 \cdot 19$ & 0.038 & 4 & 0.51 & 0.044 \\
\hline DS & 0.64 & 0.049 & 41 & 0.45 & 0.039 & 20 & 0.39 & 0.041 \\
\hline Med-DQI & 0.85 & 0.045 & 72 & 0.02 & 0.028 & 0.04 & $0 \cdot 27$ & 0.025 \\
\hline MAI & 0.83 & 0.043 & 69 & -0.52 & 0.027 & 27 & 0.04 & 0.025 \\
\hline MDP & $0 \cdot 76$ & 0.046 & 58 & -0.35 & 0.031 & 12 & $0 \cdot 30$ & 0.027 \\
\hline rMED & $0 \cdot 80$ & 0.046 & 64 & 0.09 & 0.030 & $0 \cdot 8$ & 0.35 & 0.031 \\
\hline MSDPS & $0 \cdot 76$ & 0.046 & 53 & $-0 \cdot 37$ & 0.032 & 14 & 0.67 & 0.030 \\
\hline PREDIMED & $0 \cdot 76$ & 0.047 & 58 & $0 \cdot 17$ & 0.032 & 3 & 0.39 & 0.036 \\
\hline Cardio & 0.67 & 0.049 & 45 & 0.28 & 0.037 & 8 & 0.47 & 0.043 \\
\hline
\end{tabular}

$\beta$, standardised coefficient; $R^{2}$, reliability coefficient; variance error, standardised about measurement error; MDS, Mediterranean Diet Score; MS, Mediterranean Score; DS, Dietary Score; Med-DQI, Mediterranean-Dietary Quality Index; MAI, Mediterranean Adequacy Index; MDP, Mediterranean Dietary Pattern adherence index; rMED, relative Mediterranean diet; MSDPS, Mediterranean Style Dietary Pattern Score; PREDIMED, Mediterranean food pattern PREDIMED Study; Cardio, Cardioprotective Mediterranean diet index.

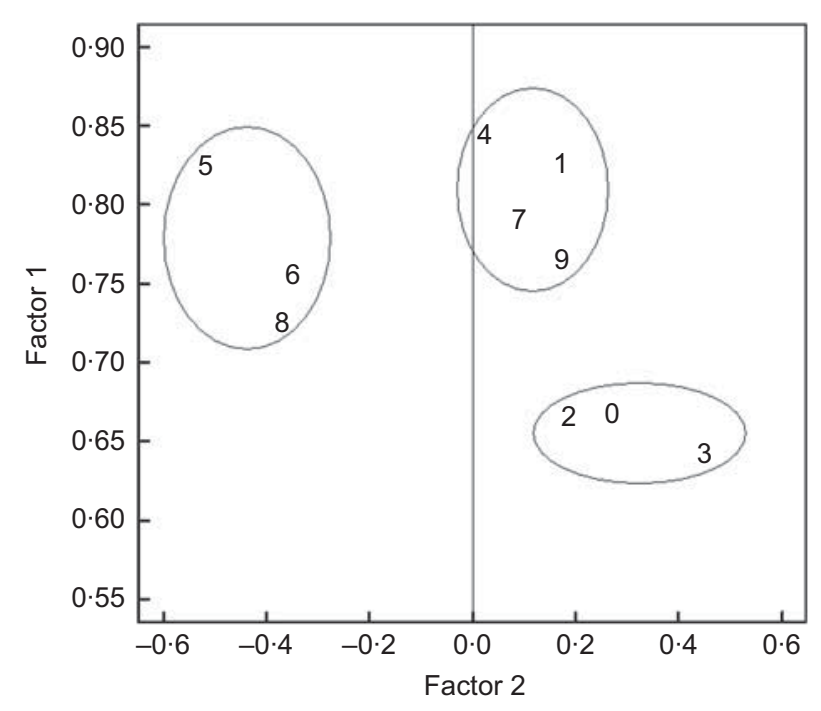

Fig. 2 Factor chart Mediterranean diet indexes (1: Mediterranean Diet Score; 2: Mediterranean Score; 3: Dietary Score; 4: Mediterranean-Dietary Quality Index; 5: Mediterranean Adequacy Index; 6: Mediterranean Dietary Pattern adherence index; 7: relative Mediterranean diet; 8: Mediterranean Style Dietary Pattern Score; 9: Mediterranean food pattern PREDIMED Study; 0: Cardioprotective Mediterranean diet index)

index is applied, high scores for adherence are frequent. Conversely, the MSDPS index generates lower values for compliance (Fig. 1). Therefore, possible associations that can be assessed between the degree of adherence to Mediterranean dietary pattern and diseases may be biased, in spite of numerous references indicating an inverse relationship ${ }^{(6,11-13,16,20)}$.

All the indexes included in the present study measure the same concept: the degree of adherence to a dietary pattern based on consumption of certain foods that are characteristic of the Mediterranean area. Hence, in theory, the correlations between these indexes should be strong; however, we found that they were weak. This weakness is probably explained by how these indexes have been developed.
For example, the indexes vary in the components included, the weight given to each and the score used.

To identify the main factors explaining this variability, we carried out an exploratory factor analysis. Much of the variability shown by the indexes was explained by two factors. The first factor explained approximately $71 \%$ of the variability, so that there was a main common factor to all indexes. Because the indexes were designed to assess the degree of adherence to the Mediterranean diet, the main factor was understood as the Mediterranean dietary pattern.

Most indexes showed correlations above 0.75 with this latent attribute (Mediterranean diet; Table 3), although it should be noted that the measurement error ranged from $28 \%$ to $59 \%\left(1-R^{2}\right.$; Table 3$)$ of the variability of the measurements, thus indicating weak to fair reliability.

The second factor explained almost $18 \%$ of the variability. Thus, reducing the dimensionality of the indexes to these two factors explained $89 \%$ of the variability, so that the model fits the data excellently.

However, the correlation between the indexes and the second factor was uneven: indexes such as MAI, MSDPS or MDP had a strong negative correlation, whereas others had a low or null correlation (rMED, MedDQI) and DS had a high positive correlation. As the first factor grouped most of the indexes into a single group because they all shared a common factor, this second factor is a divergence element between indexes because it separates indexes into three differentiated groups: negative correlation (MAI, MSDPS and MDP), low or null correlation (rMED, MedDQI, MDS, PrediMED) and high positive correlation (DS, Cardio and MS; Table 3). The most plausible hypothesis is that this second factor is related to the way in which components are treated in the index: weight or plus/minus sign of the effect of components in the score, for example.

To identify the components that are related to the second factor, we extracted factor scores for each subject and analysed the correlation between Mediterranean and non-Mediterranean components of the MAI index with 
these factor scores. A strong positive correlation was detected between factor 1 and Mediterranean food and a strong negative correlation between factor 1 and no Mediterranean food. These results reaffirm the hypothesis that this factor corresponds to the latent attribute 'Mediterranean dietary pattern'. In contrast, only the second factor showed correlation with non-Mediterranean food, mainly foodstuff of animal origin such as meat and dairy products.

We also evaluated the correlation of the food groups most commonly used in indexes (and typically Mediterranean) and the two factors to observe in more detail the associations between factors and food groups. A strong positive correlation between the second factor and dairy products and lean meat (poultry and game meat) was observed, whereas a strong negative correlation was found with ratio of fatty acids (MS). Therefore, these food groups were identified as related to the second factor and thus a source of divergence between the indexes. A reason for this could be that these components are involved in different ways in each index. To corroborate our hypothesis, the factor analysis was repeated excluding dairy and lean meat (MS ratio was maintained because it also had a strong correlation with factor 1) and the variability explained by one factor increased from $71 \%$ to almost $82 \%$ (data not shown). These observations imply that these indexes are more similar when these components are excluded. This finding confirms the presence of this second factor as a source of disagreement among the indexes. Although the Mediterranean dietary pattern has been defined, ambiguities remain to be solved ${ }^{(20,38)}$.

On the other hand, there are few studies assessing the external validity and reliability of the Mediterranean diet indexes. Perhaps, this is due to the fact that research about validity and reliability has mainly been focused on the FFQ validity ${ }^{(39)}$. However, the findings of some recent works are along the same lines as those shown here ${ }^{(40,41)}$.

One of the main concerns is the lack of agreement on the definition of the Mediterranean diet. Thus, the components included in a Mediterranean pattern adherence index can vary depending on the criteria applied; consequently, the reliability of the indexes can be dramatically lowered. Therefore, the contribution of each component in the indexes, the number of components and the scoring criteria should be established in order to improve the reliability and concordance between the indexes. Thus, by improving these indexes the higher correlations will lead to stronger evidence of the inverse relation between the Mediterranean dietary pattern and the prevalence of several diseases, such as cancer, CVD or obesity.

Given the heterogeneity of the Mediterranean diet across several countries, we recommend further confirmatory factor analysis on these populations in order to test the validity of the results reported here.

In general, the indexes performed satisfactorily in measuring adherence to the Mediterranean diet. However, the lack of high correlation between them indicates 
the need to reach a consensus on the components included in the Mediterranean diet indexes, specifically the dairy products, MS ratio and lean meat.

On the basis of the correlations observed between the main factor and the components, we conclude that, in addition to the MS ratio, fruit and vegetables are the most correlated components of indexes designed to assess adherence to the Mediterranean diet. In addition, as expected, we also found strong positive correlations between adherence to the Mediterranean diet and olive oil, pulses, cereals and fish components; on the other hand, a strong negative correlation was found with red meat. Thus, these components should be included in a reliable index to assess adherence to the Mediterranean diet, so that the index would make easy the discrimination of the consumption among subjects increasing the internal consistency. Conversely, weak correlations were found between the adherence to the Mediterranean diet and potatoes, alcohol, dairy products and lean meat.

However, given that different correlations between components and the adherence to the Mediterranean diet have been found, the weights of the components in such indexes should be adjusted accordingly.

\section{Acknowledgements}

None of the authors had any financial interest or support for this paper. The authors have no conflict of interest to declare. The authors contributed as follows: R.M.V.: study concept and design, analysis and interpretation of data, drafting of the manuscript; A.B.F.: study concept and design, drafting of the manuscript; J.P.: study concept and design, statistical analysis of data; A.P.: acquisition of data, drafting of the manuscript; A.F.: acquisition of data, drafting of the manuscript; L.S.M.: analysis and interpretation of data, drafting of the manuscript; J.L.C.: study supervision, study concept and design, analysis and interpretation of data, drafting of the manuscript. All authors have seen and approved the final version of the manuscript. The authors acknowledge the valuable assistance provided for data collection by Centre d'Ensenyament Superior de Nutrició i Dietètica (CESNID) from University of Barcelona.

\section{References}

1. Keys A \& Grande F (1957) Role of dietary fat in human nutrition. III. Diet and the epidemiology of coronary heart disease. Am J Public Health Nations Health 47, 1520-1530.

2. Willet W, Sacks F, Trichopoulou A et al. (1995) Mediterranean diet pyramid: a cultural model for healthy eating. Am J Clin Nutr 61, Suppl. 6, 1402S-1406S.

3. Matelas A, Zampelas A, Stavrinos V et al. (2000) The Mediterranean Diet: Constituents and Health Promotion. Boca Raton, FL: CRC Press.

4. González CA, Argilaga S, Agudo A et al. (2002) Diferencias sociodemográficas en la adhesión al patrón de dieta mediterránea en poblaciones de España. Gaceta Sanitaria 16, 214-221.

5. Serra-Majem L, Bach A \& Roman B (2006) Recognition of the Mediterranean diet: going a step further. Public Health Nutr 9, 101-102.

6. Sofi F, Cesari F, Abbate R et al. (2008) Adherence to Mediterranean diet and health status: meta-analysis. BMJ 337, a1344.

7. Trichopoulou A, Kouris-Blazos A, Wahlqvist ML et al. (1995) Diet and overall survival in elderly people. BMJ 311, 1457-1460.

8. Trichopoulou A, Costacou T, Bamia C et al. (2003) Adherence to a Mediterranean and survival in a Greek population. N Engl J Med 348, 2599-2608.

9. Bamia C, Trichopoulos D, Ferrari P et al. (2007) Dietary patterns and survival of older Europeans: the EPIC-Elderly Study (European Prospective Investigation into Cancer and Nutrition). Public Health Nutr 10, 590-598.

10. Bach A, Serra-Majem L, Carrasco JL et al. (2006) The use of indexes evaluating the adherence to the Mediterranean diet in epidemiological studies: a review. Public Health Nutr $\mathbf{9}$, 132-146.

11. Trichopoulou A, Bamia C \& Trichopoulos D (2009) Anatomy of health effects of Mediterranean diet: Greek EPIC prospective cohort study. BMJ 338, b2337.

12. Martínez-González MA, García-López M, Bes-Rastrollo M et al. (2011) Mediterranean diet and the incidence of cardiovascular disease: a Spanish cohort. Nutr Metab Cardiovasc Dis 21, 437-444.

13. Bibiloni M, Martínez E, Llull $\mathrm{R}$ et al. (2011) Metabolic syndrome in adolescents in the Balearic Islands, a Mediterranean region. Nutr Metab Cardiovasc Dis 21, 446-454.

14. Martínez-González MA, Fernández-Jarne E \& Serrano Martínez M (2002) Mediterranean diet and reduction in the risk of a first acute myocardial infarction: an operational healthy dietary score. Eur J Nutr 41, 153-160.

15. Buckland G, Agudo A, Luján L et al. (2010) Adherence to a Mediterranean diet and risk of gastric adenocarcinoma within the European Prospective Investigation into Cancer and Nutrition (EPIC) cohort study. Am J Clin Nutr 91, 381-390.

16. La Vecchia C \& Bosetti C (2006) Diet and cancer risk in Mediterranean countries: open issues. Public Health Nutr 9, 1077-1082.

17. Toledo E, de A Carmona-Torre F, Alonso A et al. (2010) Hypothesis-oriented food patterns and incidence of hypertension: 6-year follow-up of the SUN (Seguimiento Universidad de Navarra) prospective cohort. Public Health Nutr 13, 338-349.

18. Romaguera D, Norat T, Mouw T et al. (2009) Adherence to the Mediterranean diet is associated with lower abdominal adiposity in European men and women. J Nutr 139, 1728-1737.

19. Yannakoulia M, Panagiotakos D, Pitsavos C et al. (2009) Five-year incidence of obesity and its determinants: the ATTICA study. Public Health Nutr 12, 36-43.

20. Martínez-González MA, de la Fuente-Arrillaga C, NunezCordoba JM et al. (2008) Adherence to Mediterranean diet and risk of developing diabetes: prospective cohort study. BMJ 336, 1348-1351.

21. Waijers PM, Feskens EJ \& Ocke MC (2007) A critical review of predefined diet quality scores. BMJ 97, 219-231.

22. Van Dam RM (2005) New approaches to the study of dietary patterns. Br J Nutr 93, 573-574.

23. Serra Majem L, Ribas L, García Closas R et al. (1996) Llibre Blanc: Avaluació de l'estat nutricional de la població catalana (1992-93). Barcelona: Departament de Sanitat i Seguretat Social.

24. Farran A, Zamora R \& Cervera P (2004) Food Composition Tables of CESNID (The Centre for Superior Studies in Nutrition 
and Dietetics). Barcelona: McGraw-Hill/Interamericana de España, S.A.U. Ediciones Universitat de Barcelona.

25. Trichopoulou A, Orfanos P, Norat T et al. (2005) Modified Mediterranean diet and survival: EPIC-elderly prospective cohort study. BMJ 330, 991-997.

26. Goulet J, Lamarche B \& Nadeau G (2003) Effect of a nutritional intervention promoting the Mediterranean food pattern on plasma lipids, lipoproteins and body weight in healthy FrenchCanadian women. Atherosclerosis 170, 115-124.

27. Panagiotakos D, Pitsavos C \& Polychronopoulos E (2004) Can a Mediterranean diet moderate the development and clinical progression of coronary heart disease? A systematic review. Med Sci Monit 10, 193-198.

28. Gerber M (2006) Qualitative methods to evaluate Mediterranean diet in adults. Public Health Nutr 9, 147-151.

29. Sanchez-Villegas A, Martinez J, de Irala J et al. (2002) Determinants of the adherence to an 'a priori' defined Mediterranean dietary pattern. Eur J Nutr 41, 249-257.

30. Alberti-Fidanza A, Fidanza F, Chiuchiu M et al. (1999) Dietary studies on two rural Italian population groups of the Seven Countries Study. 3. Trend of food and nutrient intake from 1960 to 1991. Eur J Clin Nutr 53, 854-860.

31. Marcella E, Rumawas JT, Dwyer M et al. (2009) The development of the Mediterranean-style dietary pattern score and its application to the American diet in the Framingham Offspring Cohort. J Nutr 139, 1150-1156.

32. Sanchez-Tainta A, Estruch R, Bullo M et al. (2008) Adherence to a Mediterranean-type diet and reduced prevalence of clustered cardiovascular risk factors in a cohort of 3204 high-risk patients. Eur J Cardiovasc Prev Rehab 15, 589-593.

33. Buckland G, Gonzalez CA, Agudo A et al. (2009) Adherence to the Mediterranean diet and risk of coronary heart disease in the Spanish EPIC Cohort Study. Am J Epidemiol 170, 1518-1529.

34. Martinez-Gonzalez MA, Fernandez-Jarne E, Serrano-Martinez $\mathrm{M}$ et al. (2004) Development of a short dietary intake questionnaire for the quantitative estimation of adherence to a cardioprotective Mediterranean diet. Eur J Clin Nutr 58, $1550-1552$.

35. Brown TA (2006) Confirmatory Factor Analysis for Applied Research. New York, NY: Guilford.

36. Hu F (2002) Dietary pattern analysis: a new direction in nutritional epidemiology. Curr Opin Lipidol 13, 3-9.

37. Kant AK (2004) Dietary patterns and health outcomes. J Am Diet Assoc 104, 615-635.

38. Serra-Majem L, Trichopoulou A, Ngo J et al. (2004) Does the definition of the Mediterranean diet need to be updated? Public Health Nutr 7, 927-929.

39. Benítez-Arciniega AA, Mendez MA, Baena-Díez JM et al. (2011) Concurrent and construct validity of Mediterranean diet scores as assessed by an FFQ. Public Health Nutr 11, 1-7.

40. Alberti A, Fruttini F \& Fidanza F (2009) The Mediterranean Adequacy Index: further confirming results of validity. Nutr Metab Cardiovasc Dis 19, 61-66.

41. Panagiotakos D, Kalogeropoulos N, Pitsavos C et al. (2009) Validation of the MedDietScore via the determination of plasma fatty acids. Int J Food Sci Nutr 60, 168-180. 